\title{
Multi-observer contouring of male pelvic anatomy: highly variable agreement across conventional and emerging structures of interest on $\mathrm{CT}$ and $\mathrm{MR}$
}

Dale Roach MSc ${ }^{1,2}$, Lois C. Holloway PhD ${ }^{1,2,5,6}$, Michael G. Jameson PhD ${ }^{1,2,5,6}$, Jason A. Dowling $\mathrm{PhD}^{1,5,7,8}$, Angel Kennedy $\mathrm{BSc}^{3}$, Peter B. Greer PhD ${ }^{8,9}$, Michele Krawiec $\mathrm{BSc}^{3}$, Robba Rai MHIthSc ${ }^{1,2,6}$, Jim Denham $\mathrm{MD}^{10}$, Jeremiah De Leon MBBS ${ }^{12}$, Karen Lim MBBS, FRANZCR ${ }^{6,13}$, Megan E. Berry $M_{B B S}^{6,13}$, Rohen T. White MBBS ${ }^{3}$, Sean A. Bydder MBA, MPH ${ }^{3}$, Hendrick T. Tan MBBS $S^{3}$, Jeremy D. Croker MBBS ${ }^{11}$, Alycea McGrath $\mathrm{MBBS}^{3}$, John Matthews $\mathrm{MBBS}^{14}$, Robert J. Smeenk MD, PhD ${ }^{15}$, Martin A. Ebert PhD $\mathrm{P}^{3,4,5}$

${ }^{1}$ Faculty of Medicine, University of New South Wales, Sydney, Australia

${ }^{2}$ Ingham Institute for Applied Medical Research, Sydney, Australia

${ }^{3}$ Radiation Oncology, Sir Charles Gairdner Hospital, Nedlands, Australia

${ }^{4}$ School of Physics and Astrophysics, Faculty of Science, University of Western Australia, Crawley,

Australia

${ }^{5}$ Centre for Medical Radiation Physics, University of Wollongong, Wollongong, Australia

${ }^{6}$ Department of Radiation Oncology, Liverpool and Macarthur Cancer Therapy Centres, NSW, Australia

${ }^{7}$ Australian e-Health Research Centre, CSIRO, Royal Brisbane Hospital, Australia

${ }^{8}$ School of Mathematical and Physical Sciences, University of Newcastle, Newcastle, Australia

${ }^{9}$ Calvary Mater Newcastle Hospital, Newcastle, Australia

${ }^{10}$ School of Medicine and Population Health, University of Newcastle, Newcastle, Australia

${ }^{11}$ Fiona Stanley Hospital, Murdoch, Australia

${ }^{12}$ Illawarra Cancer Care Centre, Wollongong, Australia

${ }^{13}$ South Western Sydney Clinical School, University of New South Wales, Sydney, NSW, Australia

${ }^{14}$ Radiation Oncology, Auckland City Hospital, Auckland, New Zealand

${ }^{15}$ Department of Radiation Oncology, Radboud University Medical Centre, Nijmegen, The Netherlands

\section{Corresponding Author:}

Dale Roach: d.roach@student.unsw.edu.au

Running Title:

Contouring variation male pelvis structures

Conflict of Interest:

None

Acknowledgements:

The project was funded by NHMRC project grant number 1077788 


\section{Conflicts of Interest Notification}

None 


\begin{abstract}
Purpose: To quantify contouring variation amongst clinicians for multiple male pelvic structures relevant to prostate cancer radiotherapy progression and toxicity response studies.

Methods and Materials: Five prostate cancer patient datasets (CT and T2-weighted MR) were distributed to thirteen participants. Guidelines for contouring were circulated with expert-defined contours on a sample patient. Structures contoured on CT included clinical target volume (CTV), seminal vesicles, rectum, distal colon, bowel bag, bladder, and peri-rectal space (PRS). MR structures contoured were CTV, trigone, membranous urethra, penile bulb, and various neurovascular and muscular structures. Contouring variations were assessed using intraclass correlation coefficient (ICC), Dice similarity coefficient (DSC), and multiple additional metrics.
\end{abstract}

Results: CTV (CT and MR), bladder, rectum, and PRS showed excellent agreement between observer contours assessed via correlation $(I C C=0.97,0.90-1.00 ; 0.99,0.98-1.00 ; 1.00,1.00-1.00 ; 0.95$, $0.85-0.99 ; 0.90,0.69-0.99$ respectively) and overlap (DSC $=0.83 \pm 0.05 ; 0.88 \pm 0.05 ; 0.93 \pm 0.03$; $0.81 \pm 0.07 ; 0.80 \pm 0.06$ ). Seminal vesicles displayed more variation (ICC $=0.75,0.36-0.97 ; \mathrm{DSC}=$ $0.73 \pm 0.14)$, while distal colon and bowel bag showed excellent volume agreement $($ ICC $=0.97,0.92$ $-1.00 ; 0.97,0.75-1.00$ ) but poor overlap ( $\mathrm{DSC}=0.58 \pm 0.22 ; 0.67 \pm 0.21$ ). Smaller pelvic substructures displayed significant contouring variations (ICC $=0.30-0.97, \mathrm{DSC}=0.15-0.66$ ). Wilcoxon analysis revealed significant differences $(p<0.05)$ between CT and MR CTV contours across all five patients.

Conclusions: Contouring variation amongst clinicians is significant for multiple pelvic structures. This must be considered when applying derived models and constraints as future trials explore toxicity relationships, and as treatment planning applies those relationships, for both established and potential organs-at-risk.

\title{
Introduction
}

Uncertainties in contouring are one of the largest contributors to poor quality radiotherapy [1]. Quality assurance investigations of large trials have revealed contouring to be poor, requiring amendments in over $80 \%$ of audited cases [2,3]. Non-compliant contouring has been shown to impact treatment outcome in patients during clinical trials $[4,5]$.

Inter-observer contouring variations for prostate, seminal vesicles, rectum, and bladder have been investigated [6], with contouring guidelines and training significantly reducing observer variability [79]. Additionally, clinical target volumes (CTVs) contoured on magnetic resonance (MR) imaging show less variation than CTVs contoured on computed tomography (CT), with reduced volumes resulting from better soft tissue definition of the prostatic apex on MR $[10,11]$. Overlap metrics quantify contouring variations, with Dice similarity coefficient (DSC) values exceeding 0.7 considered clinically acceptable for prostate, bladder, and rectum contours $[12,13]$.

Growing numbers of studies are investigating dose to neighbouring prostatic structures as potential sources for toxicities following radiotherapy. Structures investigated include penile bulb and neurovascular bundle [14, 15], trigone [16], peri-rectal space [17], and various pelvic floor muscles [18]. However, within these studies contouring was performed either by only a single expert, defined by simple geometric borders, or no information on the contouring process was provided. As poorquality contouring could potentially mask findings from clinical trials [4], and radiotherapy treatment planning relies on dose-volume information which is progressively refined for more diverse anatomy, it is important to assess observer contouring variations for these structures. This study 
aimed to document and quantify inter-observer contouring variations across multiple male pelvic sub-structures on $\mathrm{CT}$ and MR.

\section{Methods and Materials}

\section{Patient selection}

Five prostate cancer patients were selected based on prior clustering work for a larger retrospective atlas segmentation study $[19,20]$. Using deformable registration and hierarchical clustering, these 5 patients were determined to best represent the diversity in anatomical information present in a large multi-centre clinical trial dataset [21]. Each patient dataset included co-registered whole pelvis planning-CT and a small field-of-view T2-weighted MR scan [22]. Patient datasets were distributed to 13 observers ( 9 radiation oncologists, 2 medical physicists, 1 radiotherapist, 1 radiographer) across 5 centres. Clinical experience level ranged from highly experienced to specialist trainees.

\section{Contouring}

Contoured structures for this study are listed in Table 1. The imaging modality utilised for contouring each structure was pre-determined based on expert genitourinary radiation oncologist advice for concurrent atlas-based investigations. Study guidelines containing expert defined contours derived from outsourced clinicians delineated on a sample patient were distributed to all observers (supplementary material). The CT and MR sample patient scans and contours were distributed in DICOM format to observers for additional reference. Contouring was completed on treatment planning systems available within the observer's institution, with additional contour post-processing detailed within supplementary material.

\section{Interobserver variability assessment}

DICOM structure files were converted to NifTI format for analysis within MilxView, an open-sourced image manipulation and processing platform developed by the Commonwealth Scientific and Industrial Research Organisation (CSIRO) [23]. Intraclass correlation coefficients (ICC) for contoured volumes were calculated for all structures, with ICC $>0.75$ considered excellent inter-observer agreement [24]. Variations amongst observer's contours were assessed pairwise using contouring variation metrics DSC, volume similarity, sensitivity, specificity, $95^{\text {th }}$ percentile Hausdorff distance (95_HAUS), mean absolute surface distance (MASD), and Euclidean centroid distance (Table e1) [25].

Table 1: Contoured structures

\begin{tabular}{|l|l|}
\hline CT Structures & MR Structures \\
\hline Prostate (CTV) & Prostate (CTV) \\
\hline Seminal vesicles (SV) & Trigone \\
\hline Rectum & Membranous urethra (Memb. urethra) \\
\hline Distal colon & Penile bulb \\
\hline Bowel bag & Neurovascular bundle left (NVB left) \\
\hline Bladder & Neurovascular bundle right (NVB right) \\
\hline Peri-rectal space (PRS) & Internal anal sphincter (IAS) \\
\hline & External anal sphincter (EAS) \\
\hline & Puborectalis muscle (PRM) \\
\hline & Levator ani muscles (LAM) \\
\hline
\end{tabular}




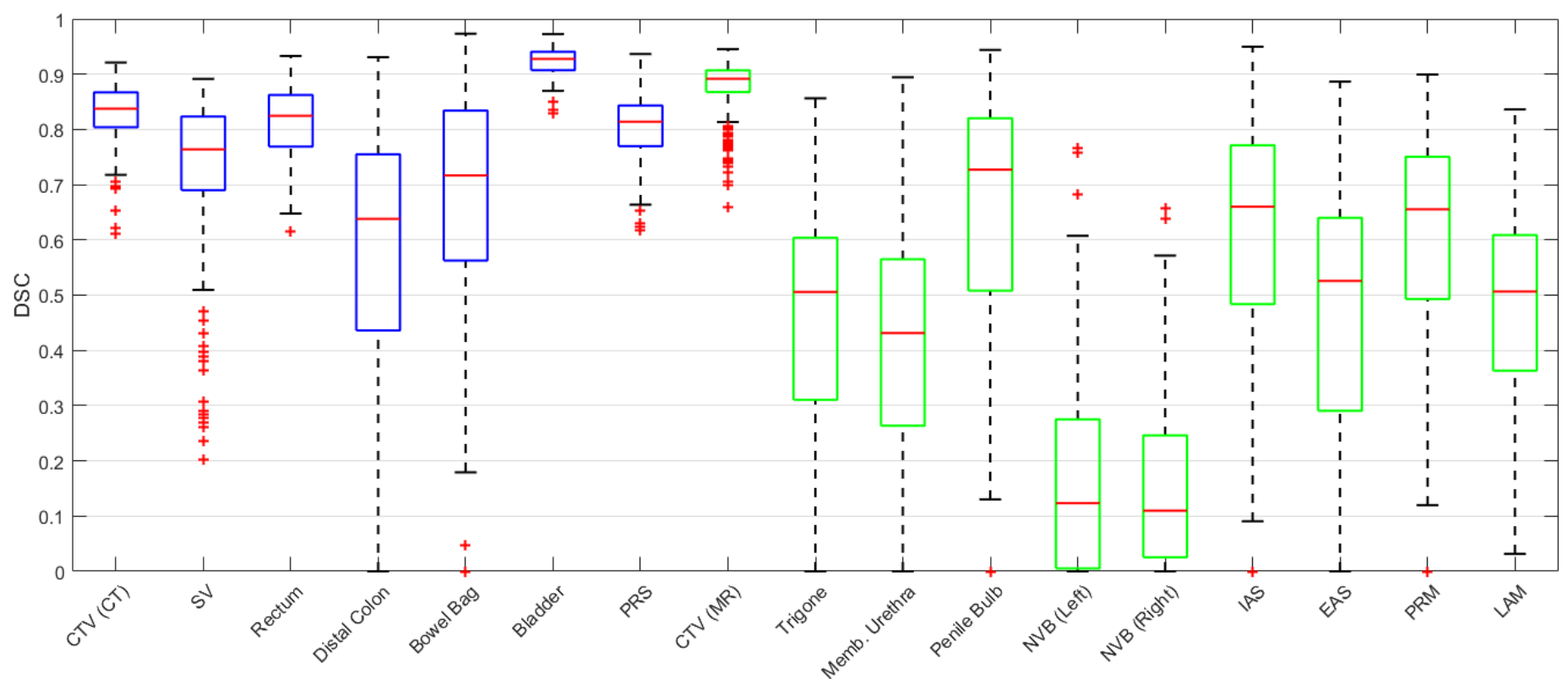

Figure 1: Pairwise DSC for CT (blue) and MR (green) structures (see Table 1 for acronym definitions)

\section{Statistics}

Differences between CT and MR CTV volumes were assessed using two-sided Wilcoxon rank sum testing ( $p<0.05$ considered significant).

\section{Results}

Mean, standard deviation, and coefficient of variation (COV) of volumes for all structures are listed in Table 2. Pairwise DSC are graphed in Figure 1, with additional statistics given in Table e2. Volume similarity, sensitivity, specificity, 95_HAUS, MASD, and Euclidean centroid distance values are compiled within the supplementary material (Figures e2 - e6, Tables e3 - e8).

\section{CTV}

Observer CTV volumes showed excellent agreement across CT (ICC $=0.97,0.90-1.00)$ and MR (ICC = $0.99,0.98-1.00$ ). Median CT volumes were larger than MR (Table 2), with Wilcoxon analysis indicating significant differences across all five patients (Figure 2). Mean 95_HAUS for MR contours were smaller than CT (Table e5, Figure e4), excluding patient 5 (MR: $10.39 \mathrm{~mm}, \mathrm{CT}: 9.48 \mathrm{~mm}$ ). Mean pairwise DSC across all patients for CT and MR were $0.83 \pm 0.05$ and $0.88 \pm 0.05$ respectively.

\section{Seminal Vesicles}

Seminal vesicle volume agreement was moderate (ICC $=0.75,0.36-0.97)$, with reduced ICC attributed to a single observer contouring significantly larger volumes for patients 2,3 and 4 (COV = $0.58,0,41,0.86$ respectively). These volumes correspond to the outliers in DSC (Figure 1 ), volume similarity, sensitivity, 95_HAUS, and MASD (Figures e1, e2, e3, e5). Mean DSC across all patients was $0.73 \pm 0.14$.

\section{Rectum}

Excellent observer agreement in contoured rectum volumes and overlap was observed (ICC $=0.95$, $0.85-0.99 ; \mathrm{DSC}=0.81 \pm 0.07$ ). However, substantial variation in 95_HAUS existed, with a maximum 
of $59.88 \mathrm{~mm}$ recorded for patient 2 (Figure e4, Table e6). Figure 3A illustrates this, where significantly different slices for the superior rectum contour boundary were selected by the two observers. Significant variation amongst observers across all patients was observed at this boundary (Figure 3B).

\section{Distal Colon and Bowel Bag}

Distal colon and bowel bag were contoured $2 \mathrm{~cm}$ superiorly to the rectum (supplementary material). Due to large variations in rectum boundary contours, poor overlap between observer contours was observed (Figure 1, Figure 3A, Table e2). Mean DSC for distal colon and bowel bag were $0.58 \pm 0.22$ and $0.67 \pm 0.21$ respectively. Large variations in Euclidean centroid distances were observed (Figure e6). ICC calculations revealed high conformity of contoured volumes for distal colon (ICC $=0.97,0.92$ $-1.00)$ and bowel bag (ICC $=0.97,0.75-1.00)$.

\section{Bladder}

Bladder contours showed excellent agreement across volume (ICC $=1.00,1.00-1.00$ ), overlap (DSC $=0.93 \pm 0.03$ ), and MASD (MASD $=0.76 \mathrm{~mm}-1.44 \mathrm{~mm}$ ). Volume varied significantly between patients $\left(69.27 \mathrm{~cm}^{3}-400.13 \mathrm{~cm}^{3}\right)$. Observer 95_HAUS distances revealed significant outliers for patient 4 (Figure e4, Table e6), where a single observer recorded pairwise 95_HAUS distances of $72.20 \mathrm{~mm}-75.65 \mathrm{~mm}$. Inspection revealed a single slice where a thin structure had been erroneously contoured as bladder.

\section{Peri-rectal Space}

Excellent observer agreement $($ ICC $=0.90,0.69-0.99)$ with acceptable overlap $($ DSC $=0.80 \pm 0.06)$ was recorded. Being a larger structure (mean volume $=299.54 \mathrm{~cm}^{3}-431.37 \mathrm{~cm}^{3}$ ), boundary metrics were more sensitive to inter-observer contouring variations than overlap metrics. While MASD was less than $10 \mathrm{~mm}$ for all but one pair of contours (Figure e5), 95_HAUS conversely revealed all but one contour pair having at least $10 \mathrm{~mm}$ separation (Figure e4).

\section{Trigone}

Trigone ICC calculations returned a negative quantity (ICC $=-0.29,-2.51-0.84)$, which arose due to inter-observer contouring variation exceeding volume differences between patients. This is shown as the large spread in volume similarity (Figure e1, Table e3), where median volume similarity of 0.37 equates to a $45 \%$ increase between contoured volumes. Patient 5 had the highest agreement in observer volumes (Table 2). Localisation of the trigone was poor, with DSC and sensitivity revealing zero overlap between some contours. Mean DSC was $0.44 \pm 0.22$, with patient 5 the most conformal. This patient also had significantly reduced 95_HAUS $(9.25 \pm 5.47 \mathrm{~mm})$ and MASD $(2.13 \pm 1.88 \mathrm{~mm})$.

\section{Membranous Urethra}

Poor agreement between observer volumes was observed (ICC $=0.52,0.09-0.92)$, with Figure 1 revealing overlap between contours ranged from none to excellent (DSC $=0-0.89$ ). Mean DSC was $0.41 \pm 0.21$. All patients had poorly reproducible contours, with COV ranging between 0.49 and 0.74 (Table 2). Due to the small mean volumes $\left(0.56 \mathrm{~cm}^{3}-0.92 \mathrm{~cm}^{3}\right)$, contouring variations resulted in significant spread in volume similarity (Figure e1).

\section{Penile Bulb}

Penile bulb contours exhibited the highest reproducibility between observers amongst MR neighbouring structures, with excellent volume agreement $(I C C=0.96,0.88-1.00)$. Spread in 
contour overlap was observed (Figure 1 ), with mean DSC $=0.66 \pm 0.21$. Two patients recorded mean DSC exceeding 0.7 (Table e1).

\section{Neurovascular Bundles}

The left and right NVBs were the least consistently contoured structures in the study (ICC $=0.48$, $0.03-0.91 ; 0.30,-0.22-0.88$ respectively), with significant variation between contours across most metrics. Overlap was exceptionally poor (Figure 1), with mean DSC $=0.16 \pm 0.17$ and $0.15 \pm 0.15$ respectively. Volume differences were significant, with median volume similarity for left and right NVBs of 0.69 and 0.66 equating to approximately $100 \%$ volume differences between contours. Patient 3 recorded the highest mean overlap ( $D S C=0.28 \pm 0.15,0.24 \pm 0.14$ ), however this improved overlap had no significant difference on volume contoured (VOLSIM $=0.62 \pm 0.42,0.59 \pm 0.45$ respectively).

\section{Pelvic Floor Muscles}

EAS, PRM, and LAM recorded ICC > 0.7 (ICC $=0.86,0.51-0.98 ; 0.97,0.88-1.00 ; 0.79,0.44-0.97$ respectively), while IAS had poor volume agreement (ICC $=0.50,-0.01-0.92)$. Large variations in volume similarity for all pelvic floor muscles were observed (Table e2, Figure e1). Overlap between contours for IAS and PRM (DSC $=0.61 \pm 0.20,0.60 \pm 0.19$ ) were better than for EAS and LAM (DSC = $0.47 \pm 0.24 ; 0.48 \pm 0.17$ ). DSC exceeding 0.7 was observed for patients 1 and 3 for IAS (DSC $=0.71 \pm$ $0.12,0.72 \pm 0.10$ ). LAM displayed poorest specificity amongst all structures (Figure e3), a consequence of large volumes $\left(15.04 \mathrm{~cm}^{3}-27.63 \mathrm{~cm}^{3}\right)$ coupled with poor overlap (Figure 1).

Table 2: Mean, standard deviation (S.D.), and coefficient of variation (C.O.V.) of observer contour volumes

\begin{tabular}{|c|c|c|c|c|c|c|}
\hline & Patient & 1 & 2 & 3 & 4 & 5 \\
\hline \multirow[t]{3}{*}{ CTV (CT) } & Mean $\left(\mathrm{cm}^{3}\right)$ & 54.44 & 43.38 & 52.64 & 33.98 & 74.02 \\
\hline & S.D. $\left(\mathrm{cm}^{3}\right)$ & 6.58 & 6.13 & 7.96 & 7.12 & 11.14 \\
\hline & C.O.V. & 0.12 & 0.14 & 0.15 & 0.21 & 0.15 \\
\hline \multirow{3}{*}{$\begin{array}{l}\text { Seminal } \\
\text { Vesicles }\end{array}$} & Mean $\left(\mathrm{cm}^{3}\right)$ & 15.91 & 17.81 & 25.52 & 12.39 & 18.09 \\
\hline & S.D. $\left(\mathrm{cm}^{3}\right)$ & 2.26 & 10.28 & 10.52 & 10.65 & 3.26 \\
\hline & C.O.V. & 0.14 & 0.58 & 0.41 & 0.86 & 0.18 \\
\hline \multirow[t]{3}{*}{ Rectum } & Mean $\left(\mathrm{cm}^{3}\right)$ & 51.13 & 37.69 & 38.61 & 54.79 & 58.94 \\
\hline & S.D. $\left(\mathrm{cm}^{3}\right)$ & 6.20 & 6.81 & 2.78 & 9.95 & 6.82 \\
\hline & C.O.V. & 0.12 & 0.18 & 0.07 & 0.18 & 0.12 \\
\hline \multirow[t]{3}{*}{ Distal Colon } & Mean $\left(\mathrm{cm}^{3}\right)$ & 33.34 & 16.51 & 14.18 & 26.05 & 25.01 \\
\hline & S.D. $\left(\mathrm{cm}^{3}\right)$ & 4.79 & 3.54 & 4.02 & 3.79 & 1.82 \\
\hline & C.O.V. & 0.14 & 0.21 & 0.28 & 0.15 & 0.07 \\
\hline \multirow[t]{3}{*}{ Bowel Bag } & Mean $\left(\mathrm{cm}^{3}\right)$ & 418.35 & 500.47 & 294.71 & 591.69 & 656.08 \\
\hline & S.D. $\left(\mathrm{cm}^{3}\right)$ & 64.85 & 94.17 & 68.09 & 79.02 & 51.04 \\
\hline & C.O.V. & 0.16 & 0.19 & 0.23 & 0.13 & 0.08 \\
\hline \multirow[t]{3}{*}{ Bladder } & Mean $\left(\mathrm{cm}^{3}\right)$ & 69.27 & 144.81 & 400.13 & 142.32 & 84.91 \\
\hline & S.D. $\left(\mathrm{cm}^{3}\right)$ & 4.13 & 9.03 & 7.38 & 11.72 & 3.82 \\
\hline & C.O.V. & 0.06 & 0.06 & 0.02 & 0.08 & 0.05 \\
\hline \multirow{3}{*}{$\begin{array}{l}\text { Peri-Rectal } \\
\text { Space }\end{array}$} & Mean $\left(\mathrm{cm}^{3}\right)$ & 359.59 & 300.78 & 299.54 & 344.32 & 431.37 \\
\hline & S.D. $\left(\mathrm{cm}^{3}\right)$ & 61.08 & 59.72 & 38.91 & 58.10 & 59.15 \\
\hline & C.O.V. & 0.17 & 0.20 & 0.13 & 0.17 & 0.14 \\
\hline \multirow[t]{3}{*}{$\overline{C T V}$ (MR) } & Mean $\left(\mathrm{cm}^{3}\right)$ & 43.83 & 31.04 & 42.34 & 23.41 & 62.32 \\
\hline & S.D. $\left(\mathrm{cm}^{3}\right)$ & 3.81 & 4.31 & 3.77 & 4.92 & 5.39 \\
\hline & C.O.V. & 0.09 & 0.14 & 0.09 & 0.21 & 0.09 \\
\hline Trigone & Mean $\left(\mathrm{cm}^{3}\right)$ & 2.72 & 3.17 & 2.95 & 2.70 & 3.23 \\
\hline
\end{tabular}




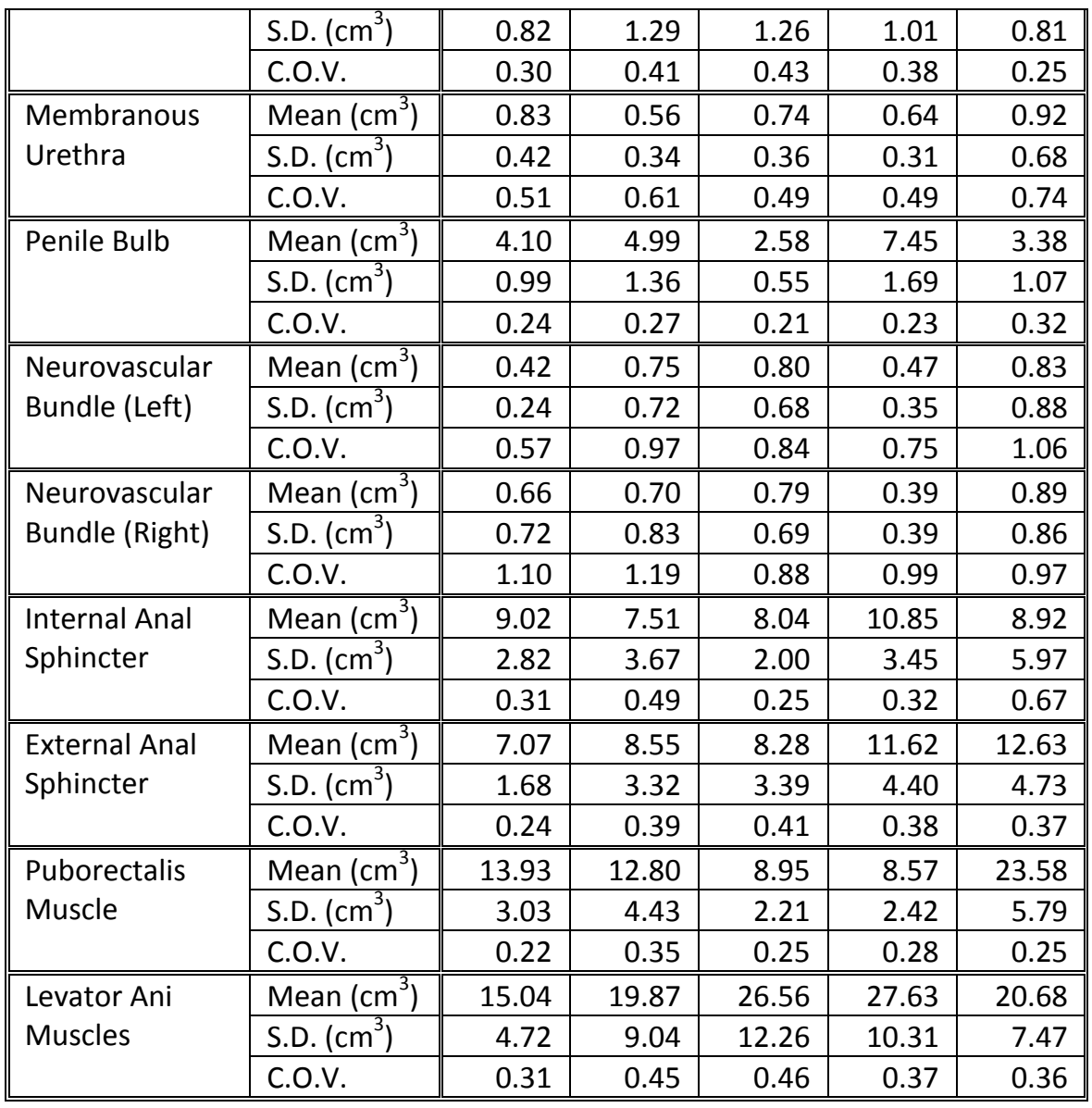

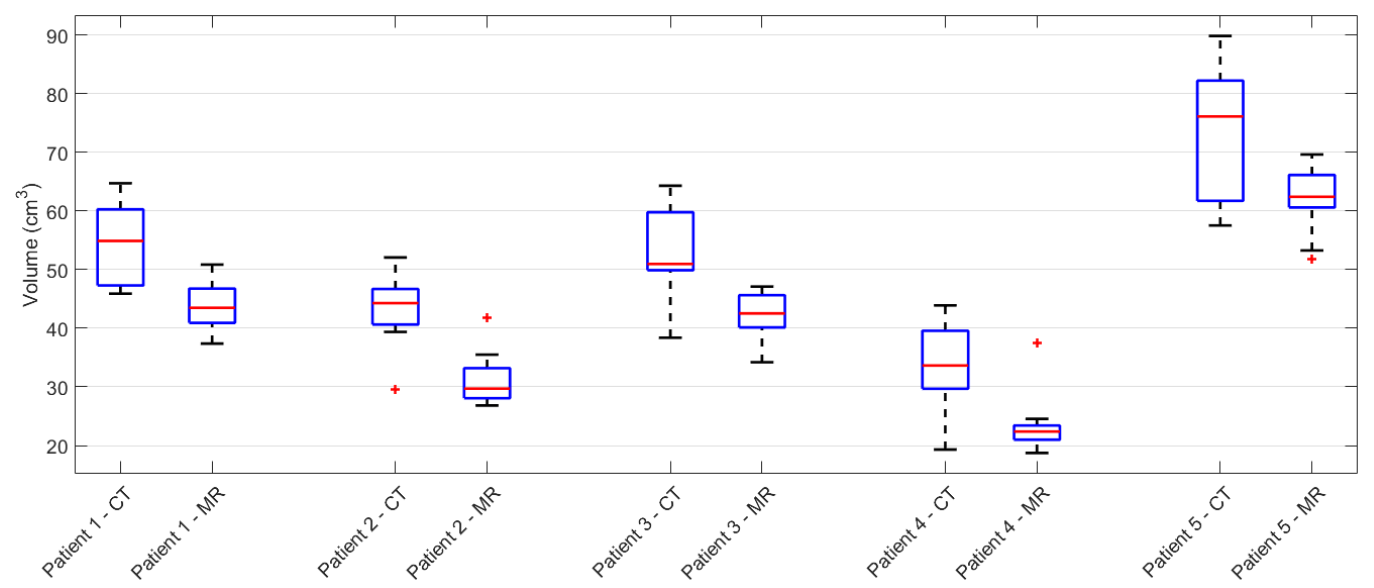

Figure 2: Observer volume variations for CT and MR defined CTV. Significant differences between median CT and MR volume were observed for all patients $(p<0.05)$.

\section{Discussion}

The study's aim was to document the range of contouring variations amongst observers for multiple male pelvic structures. These structures have established relevance to prostate cancer radiotherapy progression and toxicity response, are of emerging interest in such studies, and may be utilised in guiding radiotherapy planning optimisation. Additionally, as previous studies have shown specific contouring metrics correlating with dosimetry $[25,26]$, multiple metrics were utilised during analysis. Contouring variations for CT structures (particularly CTV, bladder, and rectum) matched 
values cited within the literature [12]. Additionally, significant reductions in CTV volumes on MR compared to $\mathrm{CT}$ (Figure 2) supports findings from prior studies investigating the utilisation of MR for prostate cancer radiotherapy $[10,11]$.

Neighbouring structures contoured on MR displayed significant inter-observer variation. This was attributed to multiple reasons. Firstly, observers within this study came from multiple institutions and professions, which prevented teaching seminars from being implemented to help reduce contouring variations [7]. As many MR structures were anatomically small in volume (Table 2), small contouring variations led to significant differences in structure localisation (Figure 4). Finally, many participants had limited experience contouring the MR structures investigated in this study. Other studies often utilised expert contours [18], which is not representative of clinical practice. This study highlights that clinician training is necessary to reduce inter-observer contouring variations for these structures.

Other studies have investigated inter-observer contouring variations for some of these structures $[27,28]$. In these investigations only a single structure was contoured, which Cassidy et al. noted would result in differing contour variations than if multiple structures were contoured simultaneously [27]. In their study, they recorded significantly improved contour overlap for NVB than was observed in this study $(I C C=0.89, D S C=0.72 \pm 0.09)$. Differences in study design include implementation of training exercises, utilisation of an expert-defined contour for 'gold-standard' analysis (opposed to pairwise analysis), and differing MR scanning sequences employed.

Additionally, screening of patients was undertaken, with patients excluded if extraprostatic extension was observed by a radiologist [27]. Within this study no screening was employed, as patient selection was based on clustering work completed for ongoing atlas analysis [20]. As only $50 \%$ of men have NVBs in standard postero-lateral positions forming distinct bundles [29], it is likely that multiple patients within this study had no identifiable NVBs. Clinicians consequently contoured NVBs using clinical knowledge rather than image intensity information derived from the patient scans, leading to the large variations in localisation of the NVBs (Figure 4).

\section{Conclusion}

Inter-observer variation for male pelvic structures contoured on CT matched variations recorded within the literature. Smaller MR structures, not regularly encountered by observers, exhibited significantly greater contouring variability. It is therefore essential that studies include contouring variation assessments amongst observers before analysis of these structures can be undertaken. 


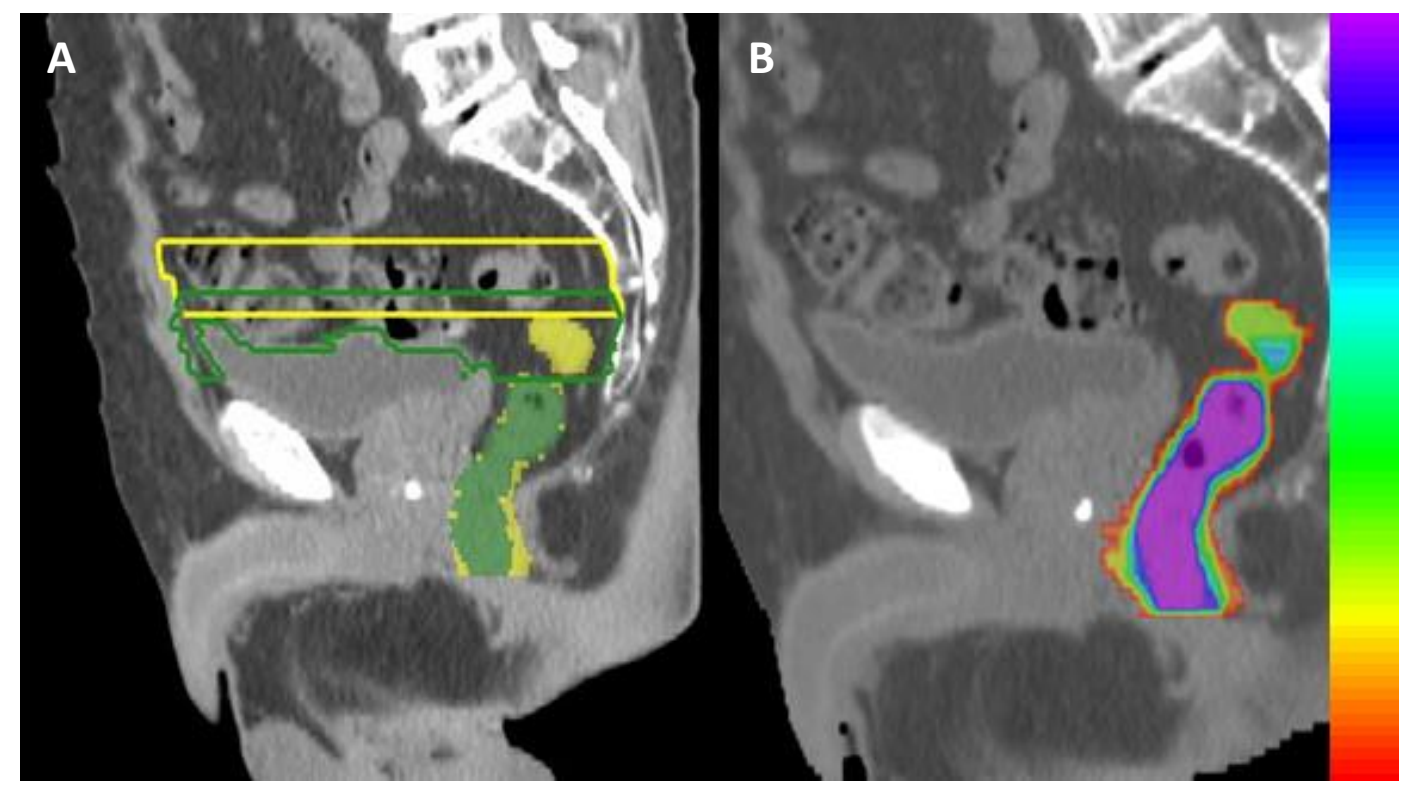

Figure 4: A) Sagittal view of patient 2, where variations in superior boundary for rectum contours between two observers (filled contours) resulted in poor overlap of bowel bag contours (outlined). B) Density distribution of all observer rectum contours for patient 2, ranging $100 \%$ observer agreement (purple) to only a single observer (red).

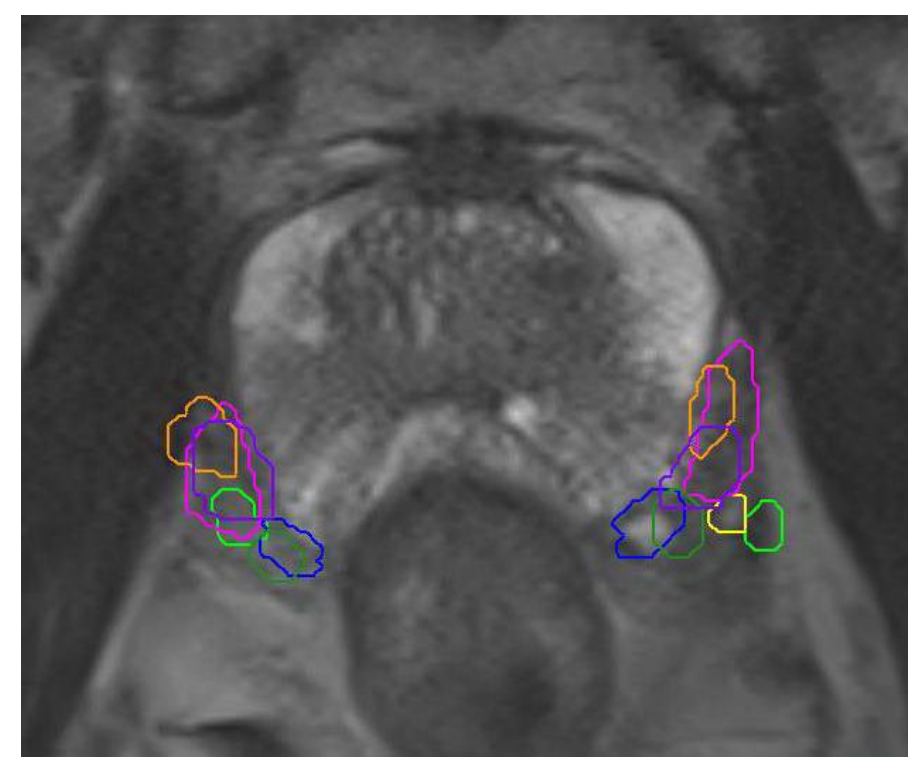

Figure 3: Variations in contouring for left and right NVB for Patient 1. For many observers, no contour overlap was evident. 


\section{References}

1. Weiss, E. and C.F. Hess, The impact of gross tumor volume (GTV) and clinical target volume (CTV) definition on the total accuracy in radiotherapy. Strahlentherapie und Onkologie, 2003. 179(1): p. 21-30.

2. Kachnic, L.A., et al., RTOG 0529: a phase 2 evaluation of dose-painted intensity modulated radiation therapy in combination with 5 -fluorouracil and mitomycin- $C$ for the reduction of acute morbidity in carcinoma of the anal canal. International Journal of Radiation Oncology* Biology* Physics, 2013. 86(1): p. 27-33.

3. Fairchild, A., et al., Quality assurance in the EORTC 22033-26033/CE5 phase III randomized trial for low grade glioma: the digital individual case review. Radiotherapy and Oncology, 2012. 103(3): p. 287-292.

4. Peters, L.J., et al., Critical impact of radiotherapy protocol compliance and quality in the treatment of advanced head and neck cancer: results from TROG 02.02. Journal of Clinical Oncology, 2010. 28(18): p. 2996-3001.

5. Abrams, R.A., et al., Failure to adhere to protocol specified radiation therapy guidelines was associated with decreased survival in RTOG 9704-a phase III trial of adjuvant chemotherapy and chemoradiotherapy for patients with resected adenocarcinoma of the pancreas. International Journal of Radiation Oncology* Biology* Physics, 2012. 82(2): p. 809-816.

6. Vinod, S.K., et al., Uncertainties in volume delineation in radiation oncology: A systematic review and recommendations for future studies. Radiotherapy and Oncology, 2016.

7. Vinod, S.K., et al., A review of interventions to reduce inter-observer variability in volume delineation in radiation oncology. Journal of medical imaging and radiation oncology, 2016. 60(3): p. 393-406.

8. Khoo, E.L., et al., Prostate contouring variation: can it be fixed? International Journal of Radiation Oncology* Biology* Physics, 2012. 82(5): p. 1923-1929.

9. Mitchell, D.M., et al., Assessing the effect of a contouring protocol on postprostatectomy radiotherapy clinical target volumes and interphysician variation. International Journal of Radiation Oncology* Biology* Physics, 2009. 75(4): p. 990-993.

10. Debois, M., et al., The contribution of magnetic resonance imaging to the three-dimensional treatment planning of localized prostate cancer. International Journal of Radiation Oncology* Biology* Physics, 1999. 45(4): p. 857-865.

11. Rasch, C., et al., Definition of the prostate in CT and MRI: a multi-observer study. International Journal of Radiation Oncology* Biology* Physics, 1999. 43(1): p. 57-66.

12. Sharp, G., et al., Vision 20/20: perspectives on automated image segmentation for radiotherapy. Medical physics, 2014. 41(5): p. 050902.

13. Dice, L.R., Measures of the amount of ecologic association between species. Ecology, 1945. 26(3): p. 297-302.

14. Mendenhall, W.M., et al., Erectile dysfunction after radiotherapy for prostate cancer. American journal of clinical oncology, 2009. 32(4): p. 443-447.

15. van der Wielen, G.J., J.P. Mulhall, and L. Incrocci, Erectile dysfunction after radiotherapy for prostate cancer and radiation dose to the penile structures: a critical review. Radiotherapy and Oncology, 2007. 84(2): p. 107-113.

16. Ghadjar, P., et al., Impact of dose to the bladder trigone on long-term urinary function after high-dose intensity modulated radiation therapy for localized prostate cancer. International Journal of Radiation Oncology* Biology* Physics, 2014. 88(2): p. 339-344.

17. Gulliford, S., et al., Radiotherapy dose-distribution to the perirectal fat space (PRS) is related to gastrointestinal control-related complications. Clinical and Translational Radiation Oncology, 2017. 7: p. 62-70.

18. Smeenk, R.J., et al., Dose-effect relationships for individual pelvic floor muscles and anorectal complaints after prostate radiotherapy. International Journal of Radiation Oncology* Biology* Physics, 2012. 83(2): p. 636-644. 
19. Dowling, J.A., et al., Automatic Substitute Computed Tomography Generation and Contouring for Magnetic Resonance Imaging (MRI)-Alone External Beam Radiation Therapy From Standard MRI Sequences. International Journal of Radiation Oncology* Biology* Physics, 2015. 93(5): p. 1144-1153.

20. Kennedy, A.M., et al., Cluster Based selection of CT exemplars from a clinical dataset for inter-patient registration and dose mapping, in International Conference on the use of Computers in Radiation Therapy. 2016: London.

21. Trans-Tasman Radiation Oncology Group (TROG), RADAR Trial - Randomised Androgen Deprivation and Radiotherapy. 2005.

22. Dowling, J.A., et al., An atlas-based electron density mapping method for magnetic resonance imaging (MRI)-alone treatment planning and adaptive MRI-based prostate radiation therapy. International Journal of Radiation Oncology* Biology* Physics, 2012. 83(1): p. e5-e11.

23. Burdett, N., et al., MILXView: a medical imaging, analysis and visualization platform, in EHealth. 2010, Springer. p. 177-186.

24. Bartko, J.J., The intraclass correlation coefficient as a measure of reliability. Psychological reports, 1966. 19(1): p. 3-11.

25. Roach, D., et al., Correlations between contouring similarity metrics and simulated treatment outcome for prostate radiotherapy. Physics in medicine and biology, 2018.

26. Jameson, M.G., et al., Correlation of contouring variation with modeled outcome for conformal non-small cell lung cancer radiotherapy. Radiotherapy and Oncology, 2014. 112(3): p. 332-336.

27. Cassidy, R.J., et al., Reproducibility in contouring the neurovascular bundle for prostate cancer radiotherapy. Practical Radiation Oncology, 2017.

28. Carillo, V., et al., Contouring variability of the penile bulb on CT images: quantitative assessment using a generalized concordance index. International Journal of Radiation Oncology* Biology* Physics, 2012. 84(3): p. 841-846.

29. Kiyoshima, K., et al., Anatomical features of periprostatic tissue and its surroundings: a histological analysis of 79 radical retropubic prostatectomy specimens. Japanese journal of clinical oncology, 2004. 34(8): p. 463-468. 